\title{
WAVE SCATTERING BY CRACKS AT MACRO- AND NANO-SCALE IN ANISOTROPIC PLANE BY BOUNDARY INTEGRAL EQUATION METHOD
}

\author{
Petia Dineva ${ }^{1}$, Tsviatko Rangelov ${ }^{2 *}$ \\ ${ }^{1}$ Institute of Mechanics, Bulgarian Academy of Sciences, Sofia 1113, Bulgaria \\ ${ }^{2}$ Institute of Mathematics and Informatics, Bulgarian Academy of Sciences, \\ Sofia 1113, Bulgaria
}

[Received 10 November 2016; Accepted 12 December 2016]

\begin{abstract}
Elastic wave scattering by cracks at macro- and nano-scale in anisotropic plane under conditions of plane strain is studied in this work. Furthermore, time-harmonic loads due to incident plane longitudinal P- or shear $\mathrm{SV}$ - wave are assumed to hold. In a subsequent step, the elastodynamic fundamental solution for general anisotropic continua derived in closed-form via the Radon transform is implemented in a numerical scheme based on the traction boundary integral equation method (BIEM). The surface elasticity effect in the case of nano-crack is taken into consideration via non-classical boundary condition along the crack surface proposed by Gurtin and Murdoch [1]. The numerical results obtained herein reveal substantial differences between anisotropic materials containing a macro- and a nano-crack in terms of their dynamic stress response, where the latter case demonstrates clearly the strong influence of the size-effects. Finally, these types of examples serve to illustrate the present approach and to show its potential for evaluating the stress concentration fields (SCF) inside cracked nanocomposites. The obtained results concern the reliability and safety of the advancing nanomaterials.
\end{abstract}

KEY WORDS: Elastodynamics, Macro/Nano-crack, Plain strain state, General anisotropy, Frequency domain, BIEM, SCF.

Math. Subj. Class. 35Q74, 74S15, 74H35

\section{STATE OF THE ART}

Modeling and evaluation of the dynamic local stress concentrations and non-uniform stress-strain distribution in advanced nanocomposite materials containing nano-defects as holes, inclusions and cracks is extremely important for the safe operation and optimum design of the engineering nanostructures subjected to dynamic loads. In the literature there exist few results for wave scattering by holes and inclusions at nano scale in elastic isotropic solids, see [2-10]. All of them, excluding $[9,10]$, propose analytical computational tools, above all, wave function expansion methods.

*Corresponding author e-mail: rangelov@ math.bas.bg 
2D elastodynamic problem for material with nano-holes and nano-inclusions under time-harmonic loads is solved by displacement BIEM in [9] for elastic isotropic infinite plane, in [10] for elastic isotropic finite solid and in [11] for elastic anisotropic finite one. The mechanical model used in all cited above papers is based on a combination of classical elastodynamic theory for the bulk solid under non-classical boundary conditions, supplemented with a localized constitutive equation for the solidinclusion interface in the frame work of Gurtin and Murdoch [1] theory of surface elasticity.

In almost all cases the nanoheterogeneities are inclusions and holes and there is a lack of results for nanocracks. The main reason is that the influence of surface effects on the fracture behaviour of materials remains unclear. The classical fracture mechanics, which neglects the effect of residual surface stress on the fracture of the materials, has been well developed. Inside its frame the surface energy is considered as a resistance to crack propagation due to the generation of new free surface. However, besides this energy absorption effect, the residual surface tension and surface elasticity which become important at nano scale would also affect the stress distributions in front of the crack tip. For nanosized crack problem, the influence of surface stress on the crack's fracture state is not well understood. There are contradictory and totally exclusive results. In [12] is discussed the limit of fracture mechanics at the nanoscale, where only a small number of atoms are included in a singular field of continuum stress formed near a crack tip. Fracture testing for mode I cracked nanoscale specimens of a brittle material as a silicon diamond-cubic structure show that the normal to the crack line stress keeps the singularity of type $1 / \sqrt{r}$ ( $r$ is the distance to the crack tip) till $0.1 \mathrm{~nm}$, although the zone of this singularity decreases with the decrease of the specimens size. However, the critical stress intensity factor (SIF) deviates from the fracture toughness near the crack tip and this evidently indicates that the fracture mechanics criterion (the crack propagates just when the SIF reaches the fracture toughness) breaks down. Or, in other words the fracture behavior of such nanoscaled specimens is no longer governed by SIF, i.e. continuum fracture behavior is not valid. In [13] is proposed a new approach for analysis of fracture through extension of continuum mechanics to the nanoscale by application of the Gurtin-Murdoch theory. As a final result of their analysis the Gurtin-Murdoch theory as applied to fracture reduces the crack-tip singularity from the square-root singularity to a logarithmic singularity.

We will shortly discuss the limited number of papers concerning mechanical problems for solids with nano-cracks.

There are some recent works studying the influence of the surface stress on the SCF near cracks, which may be regarded as asymptotic cases of voids. First Wu [14] discussed the effect of residual surface stresses on the deformation of a crack 
considering it as an elliptic void. He addresses the effect of surface stress on the static deformation of a crack, when only the constant residual surface stress is taken into consideration and deformation of the infinite thin surface layer is neglected. The assumption to neglect the surface strain is used also by other authors, see $[15,16]$. It is based on the understanding that the contribution of strain-dependent part to the surface stress is negligible compared to the residual surface tension, see [17]). However, this assumption is not correct to be used when dynamic problem is solved, as far as the boundary conditions with purely static part for the constant residual tension will not give any influence on the wave field. The residual surface tension is often ignored in the dynamic analysis.

Experiments and simulations by atomic models ( [18]) show that the stresses obtained via the atomic models are in a good agreement with the predictions of linear fracture mechanics except in a very small vicinity of the crack-tip, where the effect of surface elastic energy should be accounted for. Or, the surface elasticity effect is localized at the crack-tip.

There are series of works ( $[2,15,19-21])$ for elastostatic problems considering blunt crack with crack-tips having a small curvature radius of nanometers order via FEM. The first surface finite element was developed in [22]. In [20] was considered in-plane blunt crack with crack root presenting by a semicircular shape of radius $b$ in a finite rectangular elastic isotropic homogeneous plate subjected to uniform static tensile stress with a prescribed magnitude in a normal direction to the crack. The problem is solved by FEM with and without taking into account surface effects on the stress distribution near the crack tip fields of a mode I blunt crack. The incorporation of surface elasticity model of Gurtin and Murdoch [1] into finite element formulation is done by presenting of total potential energy as a sum of the elastic strain energy of the bulk, the elastic strain energy of the surface and the work done by the externally applied static force. The presented simulations for stresses near the crack-tip show that when the curvature radius at the crack tip reduces to nanometers, surface effects have a considerable influence on both the magnitude and positions of the maximum stresses around the crack tip. In other words, the influence of surface effect is localized in a small zone around the crack tip and it is negligible in the far field. In [21] the same approach was used but instead mode I crack a mixed-mode inclined crack in an elastic square plate was considered. In [15] is considered a crack embedded in the center of a rectangular plate subjected to uniform static tension. The crack surface is flat except in a region near the crack tip. Each crack tip is modeled as a semi-elliptical profile, and the ration of the major and the minor semi-axes of the ellipse indicates the sharpness of the crack tip.

The conclusions from the above short review are as follows: 
(a) At macroscopic scale the crack front profile is considered to be infinite sharp and the corresponding elastic and elasto-plastic near field zones are well established in classical fracture mechanics. However, in reality the crack-tips are not ideally sharp but blunt with a curvature radius in the order of microns or nanometers. Experiments and simulations by atomic models ( [18]) show that the stresses obtained via the atomic models are in a good agreement with the predictions of linear fracture mechanics except in a very small vicinity of the crack tip, where the effect of surface elastic energy should be accounted for. Or, the surface elasticity effect is localized at the crack-tip;

(b) There are a limited number of papers considering surface elasticity effects on the stresses near the crack-tip and all they are for static loads;

(c) To the authors knowledge there are no results for nano-cracks in the field of dynamic fracture mechanics even for elastic isotropic solids. For anisotropic cracked solids there are no results even for static problems.

The aim of this study is to consider the dynamic stress field near crack tip under incident time-harmonic plane $\mathrm{P}$ and SV wave in anisotropic plane by BIEM taking into account the surface elasticity effect in the frame of Gurtin and Murdoch [1] model. At macro-scale the crack is finite line crack (Fig. 1 a)), while at nano-scale a blunt crack (Fig. $1 \mathrm{~b}$ )) with crack root presenting by a semicircular shape of radius $b$ is considered.

The paper is organized as follows: the formulation of the considered in-plane elastodynamic problem for a crack at macro- and nano-scale in an anisotropic plane subjected to incident time-harmonic wave is done in Section 2, while its reformulation via boundary integral equations along existing boundaries based on the analytically derived by Radon transform fundamental solution is given in Section 3. Numerical results are shown in Section 4, and finally conclusions are in Section 5.
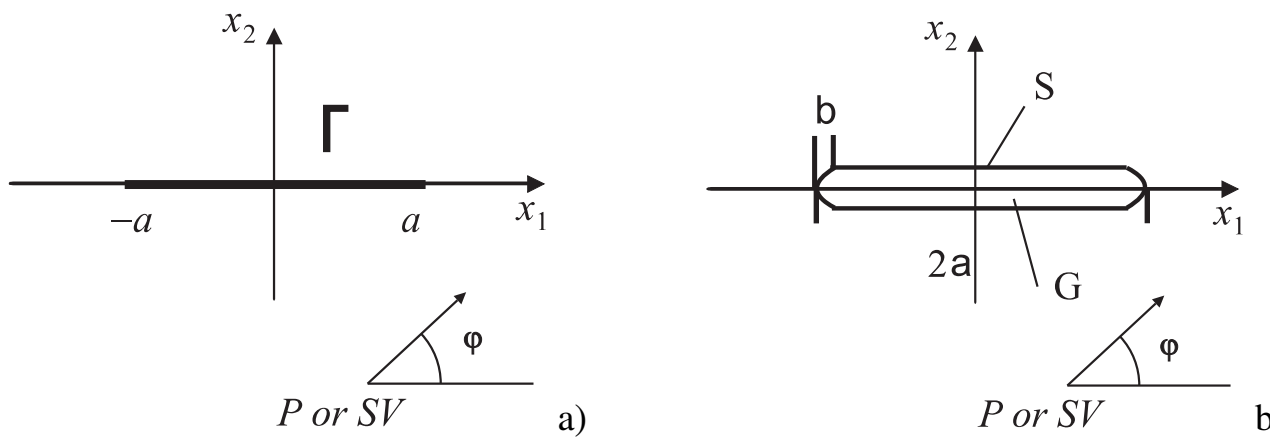

Fig. 1. The problem geometry: a) a line macro crack; b) a blunt nano-crack. Cracks are subjected to incident $\mathrm{P}$ - or SV- wave propagating in an anisotropic plane. 


\section{PROBlem FORMULATION}

In a Cartesian coordinate system $O x_{1} x_{2} x_{3}$ consider an anisotropic plane $x_{3}=0$ containing a line macro-crack $\Gamma=\Gamma^{+} \cup \Gamma^{-}$with length $|\Gamma|=2 a$ (Fig. 1 a)) or a nano-crack, a domain $G$ with boundary $S$ (Fig. 1 b)) under incident time-harmonic with frequency $\omega$ plane longitudinal $P$ or shear $S V$ wave with incident angle $\varphi$ in respect to the $O x_{1}$ coordinate axis. The nano-crack is considered as a blunt crack with crack root presenting by a semicircular shape of radius $b$ with dimension falling in the interval $10^{-7} \mathrm{~m} \div 10^{-10} \mathrm{~m}$. The crack perimeter is $|S|=2[2(a-b)+\pi b]$.

It is assumed that anisotropic material is of monoclinic type (there exists at least one elastic symmetry plane), because only under this assumption is possible uncoupling of the 3D problem into two-dimensional in-plane and anti-plane ones, see [23]. Plane strain state, i.e. in-plane wave motion in respect to plane $x_{3}=0$ is considered. In this case the only non-zero field quantities are displacements $u_{1}, u_{2}$, stresses $\sigma_{11}, \sigma_{12}, \sigma_{22}$ all dependent on coordinates $x=\left(x_{1}, x_{2}\right)$ and frequency $\omega$. What follows is to define the boundary value problem (BVP) in frequency domain by the governing elliptic partial differential equations of second order and corresponding boundary conditions.

\subsection{THE GOVERNING EQUATIONS}

The system of governing equations consists of constitutive equation, kinematic relation and equation of motion given below. In the case of general anisotropy 6 parameters $c_{11}, c_{12}, c_{16}, c_{22}, c_{26}, c_{66}$ characterize the stiffness matrix $C_{i j k l}$, where the contracted Voigt notation, see [24] $c_{p q}$ are obtained from $C_{i j k l}$ following the rule: $(11) \rightarrow 1,(22) \rightarrow 2,(12)=(21) \rightarrow 6$. Recall the symmetries for the stiffness matrix $C_{i j k l}=C_{j i k l}=C_{i j l k}=C_{k l i j}, C_{i j k l} g_{i j} g_{k l}>0$ for any non-zero real symmetric tensor $g_{i j}$. This means that the coefficients $c_{i j}$ should satisfy the following conditions for positive strain energy and real and positive wave velocities:

$c_{11}>\left|c_{12}\right|,\left(c_{11}+c_{12}\right) c_{22}>2 c_{12}^{2}, c_{66}, c_{11}>0, c_{22}>0,\left|c_{12}+c_{66}\right|<\left(\sqrt{c_{11} c_{22}}+c_{66}\right)$.

The constitutive equations - Hookes law, i.e. $\sigma_{i j}=C_{i j k l} s_{k l}$ is presented in the following matrix form:

$$
\left(\begin{array}{l}
\sigma_{11}(x, \omega) \\
\sigma_{22}(x, \omega) \\
\sigma_{12}(x, \omega)
\end{array}\right)=\left(\begin{array}{lll}
c_{11} & c_{12} & c_{16} \\
c_{12} & c_{22} & c_{26} \\
c_{16} & c_{26} & c_{66}
\end{array}\right)\left(\begin{array}{l}
s_{11}(x, \omega) \\
s_{22}(x, \omega) \\
2 s_{12}(x, \omega)
\end{array}\right)
$$

where $s_{i j}$ is the strain tensor. The orthotropic material is characterized with the following four independent material constants $c_{11}, c_{12}, c_{22}, c_{66}$, because $c_{16}=c_{26}=0$. 
For isotropic material the following relations are truth $c_{11}=c_{22}=\lambda+2 \mu$ and $c_{12}=\lambda ; c_{66}=\mu$, where $\lambda$ and $\mu$ are Lamé constants.

Under assumption of small displacements the kinematic relations are

$$
s_{i j}(x, \omega)=0.5\left[u_{i, j}(x, \omega)+u_{j, i}(x, \omega)\right], \quad i, j=1,2,
$$

where the symbol ()$_{, i}$ represents the partial derivative with respect to $x_{i}$.

In the plane $x_{3}=0$ the equation of motion is

$$
\sigma_{i j, j}(x, \omega)+\rho \omega^{2} u_{i}(x, \omega)=0, \quad x \in R^{2} \backslash \Gamma, \text { or } x \in R^{2} \backslash G,
$$

in the case of zero body force, where $\rho$ is the mass density.

\subsection{BOUNDARY CONDITIONS}

\subsubsection{BOUNDARY CONDITIONS AT MACRO-SCALE}

Along the crack line traction free boundary conditions are satisfied, i.e.

$$
t_{j}(x, \omega)=0, x \in \Gamma, \text { or } x \in S,
$$

where $t_{j}$ is the total traction, which in the case of incident plane wave is presented as a superposition of incident and scattered wave. The same is truth for the total displacement and traction fields, i.e.

$$
\begin{aligned}
& u_{i}(x, \omega)=u_{i}^{i n}(x, \omega)+u_{i}^{s c}(x, \omega), \quad x \in R^{2} \backslash \Gamma, \text { or } x \in R^{2} \backslash G, \\
& t_{i}(x, \omega)=t_{i}^{i n}(x, \omega)+t_{i}^{s c}(x, \omega), \quad x \in \Gamma, \text { or } x \in S .
\end{aligned}
$$

At infinite the Sommerfeld's radiation condition for the scattered wave is satisfied.

The BVP for the scattered wave field $u_{i}^{s c}$ consists of governing equation (2), the Sommerfeld's radiation condition at infinite and along the cracks boundary $t_{i}^{s c}=$ $-t_{i}^{i n}, x \in \Gamma$, or $x \in S$ having in mind relation (4). Thus, to solve this BVP we must know the incident wave field for any direction of propagation in the elastic anisotropic plane. Following [25], the incident wave field of plane wave propagating with frequency $\omega$ in the direction $\eta=\left(\eta_{1}, \eta_{2}\right)$ with angle $\varphi$ in respact to $O x_{1}$ axis, i.e., $\eta_{1}=\cos \varphi, \eta_{2}=\sin \varphi$ at point $x$ is as follows:

- For P-wave

$$
u_{i}^{i n}(x, \omega)=p_{i}^{1}(\eta) \exp \left[-i k_{1}(\eta)\left(x_{1} \eta_{1}+x_{2} \eta_{2}\right)\right],
$$

- For SV-wave

$$
u_{i}^{i n}(x, \omega)=p_{i}^{2}(\eta) \exp \left[-i k_{2}(\eta)\left(x_{1} \eta_{1}+x_{2} \eta_{2}\right)\right] .
$$


Here wave numbers are $k_{i}(\eta)=\omega \sqrt{\frac{\rho}{a_{i}(\eta)}}$ and $a_{i}(\eta)>0, a_{1}>a_{2}, p^{i}=\left(p_{1}^{i}, p_{2}^{i}\right)$ are eigenvalues and eigenvectors of the matrix

$$
C(\eta)=\left(\begin{array}{ll}
c_{11} \eta_{1}^{2}+c_{66} \eta_{2}^{2}+2 c_{16} \eta_{1} \eta_{2} & c_{26} \eta_{2}^{2}+\left(c_{12}+c_{66}\right) \eta_{1} \eta_{2} \\
c_{16} \eta_{1}^{2}+c_{26} \eta_{2}^{2}+\left(c_{12}+c_{66}\right) \eta_{1} \eta_{2} & c_{22} \eta_{2}^{2}+2 c_{26} \eta_{1} \eta_{2}
\end{array}\right)
$$

Note that in the isotropic case $a_{1}=\lambda+2 \mu, a_{2}=\mu$ and only in this case the eigenvalues, eigenvectors and wave numbers do not depend on $\eta$.

Once having incident displacement field, the corresponding stresses are computed by Eq. (1) and the corresponding tractions on the boundary are $t_{i}^{i n}(x, \omega)=$ $\sigma_{i j}^{i n}(x, \omega) n_{j}(x)$.

The near-field solutions for typical crack opening mode, in the frame of linear fracture mechanics, can be expressed in polar coordinates $(r, \theta)$ with the origin at the crack-tip for $r \rightarrow 0$ as, see [26]

(8) $u_{j}(r, \theta, \omega)=\sqrt{r} K_{H}(\omega) g_{j}^{H}(\theta), \quad \sigma_{i j}(r, \theta, \omega)=\frac{1}{\sqrt{r}} K_{H}(\omega) f_{i j}^{H}(\theta), H=I, I I$.

where the angular functions $g_{j}^{H}(\theta), f_{i j}^{H}(\theta)$ depend only on the material constants and $\theta$, while the coefficients $K_{I}, K_{I I}$, named stress intensity factors (SIF) depend on the frequency $\omega$. The knowledge of SIFs gives information for the strength and life time prediction of studied solid and structures. The computation of SIF is possible by the usage of well-known traction formulae. If we consider in-plane crack along the segment $A B$ with local coordinates of points $A=(-a, 0), B=(+a, 0)$ subjected to time-harmonic load, the traction formulae give:

$$
K_{I}=\lim _{x_{1} \rightarrow \pm a} \sqrt{2 \pi\left(x_{1} \mp a\right)} t_{2}, \quad K_{I I}=\lim _{x_{1} \rightarrow \pm a} \sqrt{2 \pi\left(x_{1} \mp a\right)} t_{1},
$$

where $t_{i}, i=1,2$ is the traction at a point $\left(x_{1}, 0\right)$ close to the crack-tips.

\subsubsection{BOUNDARY CONDITIONS AT NANO-SCALE}

Non-classical boundary condition for the blunt nano-crack is applied along its surface S. Note that for blunt crack the surface is (see Fig. 1 b)): $S=S^{+} \cup S^{-} \cup S^{l} \cup S^{r}$, where $S^{+}, S^{-}$are upper and lower flat part of the crack and crack-tip is presented by a semicircles $S^{l}, S^{r}$ with radius $b$ with dimension falling in the interval $10^{-7} \mathrm{~m} \div$ $10^{-10} \mathrm{~m}$.

In the frame of the [1] model the surface stress is expressed by

$$
\sigma_{i j}^{S}=\tau_{0} \delta_{i j}+\frac{\partial E}{\partial \varepsilon_{i j}^{S}} .
$$


The surface stress $\sigma_{i j}^{S}$ is related to the deformation dependent surface energy density $E$, where $\varepsilon_{i j}^{S}$ is the $2 \times 2$ strain tensor for the surface $S, \delta_{i j}$ is the Kronecker delta symbol, $\tau_{0}$ is the residual surface tension under unstrained condition (along undeformed surface) which is independent on deformation and induce an additional static deformation. The residual surface tension $\tau_{0}$ is often ignored in the dynamic analysis. Following [1], it is assumed that the surface layer $S$ with zero thickness has different elastic properties from that of the plane. Although the plane is elastic anisotropic, we assume the surface layer $S$ is elastic and isotropic. Under assumption the infinite thin layer is isotropic with surface Lamé constants $\lambda^{S}$ and $\mu^{S}$ the following conditions are satisfied in tangential and in normal direction along $S$, i.e. in local coordinate system of normal $n$ and tangential $l$ to $\mathrm{S}$ vectors:

$$
\mid \begin{aligned}
\sigma_{n l}^{M} & =\frac{\partial \sigma_{l l}^{S}}{\partial l}+\tau_{0} \frac{\psi_{l l}^{S}}{\rho} \\
\sigma_{n n}^{M} & =-\frac{\sigma_{l l}^{S}}{b}+\tau_{0} \frac{\partial \psi_{l l}^{S}}{\partial l}, \\
\psi_{l l}^{S} & =-\frac{u_{l}}{b}+\frac{\partial u_{n}}{\partial l} .
\end{aligned}
$$

Here the first equilibrium equation in (11) is written in the tangential plane and the second equation is in the normal direction to the surface boundary $S$, the super suffix $M$ means the matrix material in the plane, $\sigma_{l l}^{S}=\tau_{0}+\left(\lambda^{S}+2 \mu^{S}\right) \varepsilon_{l l}^{S}$ is the normal stress in tangential direction expressed by the constitutive equation for elastic isotropic behavior of the surface layer, $b$ is the curvature radius of the boundary $S$, $\left(u_{n}, u_{l}\right)$ are the displacement components in the local coordinate system $(n, l)$. A coherent interface $S$ is considered perfect bonded, no slip, where the strain $\varepsilon_{i j}^{S}$ in direction $l$ is equal to the associated tangential strain in the abutting bulk matrix $\varepsilon_{i j}^{M}$, i.e. $\varepsilon_{i j}^{S}=\varepsilon_{i j}^{M}$. In this case the following condition is satisfied

$$
\varepsilon_{l l}^{S}=\frac{u_{n}}{b}+\frac{\partial u_{l}}{\partial l}
$$

The discussed above boundary conditions (10) - (13) can be reformulated in the following more compact form in respect to the tractions developed along the interface boundary, see $[9,27]$ :

$$
\left(\begin{array}{c}
t_{1}^{M} \\
t_{2}^{M}
\end{array}\right)=-\frac{\tau_{0}}{b}\left(\begin{array}{c}
n_{1} \\
n_{2}
\end{array}\right)+T^{S}\left(\begin{array}{l}
u_{1} \\
u_{2}
\end{array}\right) \text { on } S,
$$

where $T^{S}=T_{1}+\frac{\partial}{\partial l} T_{2}+\frac{\partial^{2}}{\partial l^{2}} T_{3}$ and 


$$
\begin{aligned}
T_{1} & =\frac{1}{b^{2}} N\left(\begin{array}{cc}
-\alpha_{s} & \tau_{0} b_{, l} \\
-\alpha_{s} b_{, l} & -\tau_{0}
\end{array}\right) N^{\prime}, & N & =\left(\begin{array}{cc}
n_{1} & -n_{2} \\
n_{2} & n_{1}
\end{array}\right), \\
T_{2} & =N\left(\begin{array}{cc}
0 & -\beta_{s} \\
\beta_{s} & 0
\end{array}\right) N^{\prime}, & T_{3} & =N\left(\begin{array}{cc}
\tau_{0} & 0 \\
0 & \alpha
\end{array}\right) N^{\prime} .
\end{aligned}
$$

Here $\alpha_{s}=\lambda^{S}+2 \mu^{S}, \beta_{s}=\frac{1}{b}\left(\alpha_{s}+\tau_{0}\right), n_{i}=-n_{i}^{M}, \frac{\partial}{\partial l}, \frac{\partial^{2}}{\partial l^{2}}$ are first and second tangential derivatives, $t_{k}^{M}=\sigma_{k j}^{M} n_{j}$. Note that when $\tau_{0} \stackrel{\partial}{=} 0$ and $\lambda^{S}=\mu^{S}=0$ the boundary condition (14) transforms into classical boundary condition (3) describing the traction free surface of the macro-crack.

\section{BIEM FORMULATION}

\subsection{MACRO-CRACK}

The following frequency-dependent non-hypersingular traction BIE along the crack boundary $\Gamma$ in respect to the unknown crack opening displacement (COD) of the scattered wave field defined as $\Delta u_{i}^{s c}=\left.u_{i}^{s c}\right|_{\Gamma^{+}}-\left.u_{i}^{s c}\right|_{\Gamma^{-}}$along the crack $\Gamma$ describes the BVP formulated in Section 1. Following [25] we obtain the following integrodifferential equation for $\Delta u_{i}^{s c}$ :

$$
\begin{aligned}
& t_{i}^{i n}(x, \omega)=C_{i j k l} n_{j}(x) \int_{\Gamma^{+}}\left[\left(\sigma_{p \eta k}^{*}(x, \xi, \omega) \Delta u_{p, \eta}^{s c}(\xi, \omega)\right.\right. \\
& \left.\left.-\rho \omega^{2} u_{p k}^{*}(x, \xi, \omega) \Delta u_{p}^{s c}\right) \delta_{\lambda l}-\sigma_{m \lambda k}^{*}(x, \xi, \omega) \Delta u_{m, l}^{s c}(\xi, \omega)\right] n_{\lambda}(\xi) d \xi, \quad x \in \Gamma .
\end{aligned}
$$

Here the couple $x, \xi$ presents the position vectors of the source and receiver points, $\sigma_{i j q}^{*}=C_{i j k l} u_{k q, l}^{*}, u_{k q}^{*}$ is the displacement fundamental solution of the governing equation (2), see [25], Chap. 3.4, $\delta_{i j}$ is the Kronecker symbol, all indices takes values 1 and 2, the summation convention over repeated indexes is implied, while subscript commas denote partial differentiation with respect to $\xi$.

BIE (15) is integro-differential equation with respect to the COD and all singular integrals in it converge in CPV sense, if a priori smoothness requirements $\Delta u_{i}^{s c} \in C^{1+\alpha}(\Gamma)$ are fulfilled. Once having solution for the COD, the solution for displacement and stress at any point in $R^{2} \backslash \Gamma$ can be obtained by the usage of the integral representation formulae, see [28].

$$
\begin{gathered}
u_{j}^{s c}(x, \omega)=-\int_{\Gamma^{+}} \sigma_{k i j}^{*}(x, \xi, \omega) \Delta u_{k}(\xi, \omega) n_{i} d \xi, x \notin \Gamma . \\
\sigma_{p q}^{s c}(x, \omega)=-C_{p q k l} \int_{\Gamma^{+}} \sigma_{i j k, l}^{*}(x, \xi, \omega) \Delta u_{i}(\xi, \omega) n_{j} d \xi, \quad x \notin \Gamma .
\end{gathered}
$$

The total wave field is obtained by the usage of the superposition formulae (4). 


\subsection{NANO-CRACK}

In here we use integro-differential equation for $u_{i}^{s c}$ on $S$ obtained analogous as (15), see [25], but accounting for the surface effect:

$$
\begin{aligned}
& c_{i j}\left(t_{j}^{i n}(x, \omega)-t_{j}^{M}(x, \omega)\right)=C_{i j k l} n_{j}(x) \int_{S}\left[\left(\sigma_{p \eta k}^{*}(x, \xi, \omega) u_{p, \eta}^{s c}(\xi, \omega)\right.\right. \\
& \left.\left.\quad-\rho \omega^{2} u_{p k}^{*}(x, \xi, \omega) u_{p}^{s c}\right) \delta_{\lambda l}-\sigma_{m \lambda k}^{*}(x, \xi, \omega) u_{m, l}^{s c}(\xi, \omega)\right] n_{\lambda}(\xi) d \xi, \quad x \in S
\end{aligned}
$$

where $u_{i}^{M}=u_{i}^{s c}+u_{i}^{i n}, t_{i}^{i n}+t_{i}^{s c}=t_{i}^{M}, u_{i}^{i n}, t_{i}^{i n}$ are defined with (5), $c_{i j}$ is the jump term depending on the local geometry at the source point $x$. Once having the scattered displacement along the surface $S$, the total displacement and stresses at any observer point $x \notin S$ can be obtained by the superposition formulae (3) and the integral representation formulae

$$
\begin{aligned}
u_{i}(x, \omega) & =\int_{S} t_{i j}^{*}(x, \xi, \omega) u_{j}(\xi, \omega) d \xi-\int_{S} u_{i j}^{*}(x, \xi, \omega) t_{j}(\xi, \omega) d \xi, x \notin S \\
\sigma_{m p}(x, \omega) & =\int_{S}\left(\sigma_{i j m}^{*}(x, \xi, \omega) u_{j, i}(\xi, \omega)-\rho \omega^{2} u_{j}(\xi, \omega) u_{j m}^{*}\right) \delta_{q p} n_{q} d \xi \\
& -\int_{S} \sigma_{q j m}^{*}(x, \xi, \omega) u_{j, p}(\xi, \omega) n_{q} d \xi, \quad x \notin S
\end{aligned}
$$

\section{NUMERICAL RESULTS}

\subsection{NUMERICAL SCHEME VERIFICATION}

The numerical procedure for solution of the BVP formulated via BIE (15) over line $\Gamma^{+}$for a macro-crack (Fig. 1 a) and BIE (18) over boundary $S$ for a blunt nano-crack (Fig. $1 \mathrm{~b}$ ) is based on discretization and collocation approach. After discretization of the non-hypersingular traction BIEs, overcoming of the weak and strong singularities in the integrals and satisfaction of the boundary conditions, an algebraic system of equations with respect to the unknown crack opening displacements is obtained and solved. The numerical results are obtained with Mathematica 6.0.

We use 5 boundary elements (BE) on the crack $\Gamma$, with a length $|\Gamma|=2 a, a=$ $5 \times 10^{-9} \mathrm{~m}$, where $1^{-s t}$ and $5^{-t h}$ are quarter-point crack-tip BE with the length $0.15 a$. On the blunt crack $S$, with a length $|S|=2 a[2(1-0.0375)+0.0375 \pi]=$ $2.04281 \times 10^{-8} \mathrm{~m}$ we use 10 ordinary BE: 8 on $S^{-} \cup S^{+}$and 2 on semi-circle $S^{l}, S^{r}$ with radius $b=0.0375 a$.

It is used dimensionless surface parameter defined as $m=\frac{\alpha_{s}}{2 \mu b b_{m}}$ with $\alpha_{s}=$ $6.091 \mathrm{~N} / \mathrm{m}$ where in the numerical results we get $b_{m}=1,5,25,125, \infty$. Note that the case $m=0$ corresponds to the blunt crack without surface effect. In all illustrative examples the incident $\mathrm{P}$ wave propagates normal to $O x_{1}$ axis, i.e. $\varphi=\pi / 2$, 
while incident SV wave propagates with incident angle $\varphi=\pi / 3$. Also is inserted non-dimensional frequency defined as $\Omega=a \omega \sqrt{\rho / c_{22}}$. The values of $\alpha_{s}$ are taken from the literature usually determined by the atomic simulations [17] and they are in the neighbourhood of $\pm 10 \mathrm{~N} / \mathrm{m}$, where negative values are also possible.

The normalized SCF near the right crack-tip of $\Gamma^{+}$and close to $S$ at the point $(a, 0)$ is evaluating using the formulae, see (9)

$$
\begin{aligned}
& K_{I}\left(\left(x_{1}, 0\right), \omega\right)=\frac{\sigma_{22}\left(\left(x_{1}, 0\right), \omega\right)}{\sigma_{22}^{i n}\left(\left(x_{1}, 0\right), \omega\right)} \sqrt{2 \pi\left(x_{1}-a\right)}, \quad x_{1}>a, \\
& K_{I I}\left(\left(x_{1}, 0\right), \omega\right)=\frac{\sigma_{12}\left(\left(x_{1}, 0\right), \omega\right)}{\sigma_{22}^{i n}\left(\left(x_{1}, 0\right), \omega\right)} \sqrt{2 \pi\left(x_{1}-a\right)}, \quad x_{1}>a .
\end{aligned}
$$

Normalized SIF on the right crack-tip of $\Gamma$ are

$$
K_{I}(\omega)=\lim _{x_{1} \rightarrow a} K_{I}\left(\left(x_{1}, 0\right), \omega\right), \quad K_{I I}(\omega)=\lim _{x_{1} \rightarrow a} K_{I I}\left(\left(x_{1}, 0\right), \omega\right) .
$$

The BIEM formulation of the defined in Section 2. BVP is general and it allows solving dynamic fracture problems for general anisotropic cracked materials. To the authors best knowledge, there are no available results in the literature for anisotropic material with nano-cracks subjected to dynamic or even static loads. In order to verify the present approach we will compare our BIEM results for blunt nano-crack at zero surface elasticity parameter $m=0$ with the authors results for macro-crack and with available in the literature solutions for macro-crack in the case of elastic isotropic and orthotropic materials. Material parameters $c_{i j}$ are for orthotropic material, see [29], given as a ratio of $c$, i.e. $c_{i j}=\alpha_{i j} c, c=6.6495 G P a$. The density is $\rho=2.7 \times 10^{3} \mathrm{~kg}$. Following [29], three types of materials are considered:

case a) isotropic material with $\alpha_{11}=1, \alpha_{12}=1 / 3, \alpha_{22}=1, \alpha_{66}=1 / 3$;

case b) orthotropic material with $\alpha_{11}=1, \alpha_{12}=1 / 3, \alpha_{22}=1, \alpha_{66}=1 / 6$;

case c) orthotropic material with $\alpha_{11}=1, \alpha_{12}=1 / 30, \alpha_{22}=1, \alpha_{66}=1 / 3$.

The authors BIEM results for a line macro-crack (Fig. 1 a) and for a blunt nanocrack (Fig. 1 b) at surface parameter $m=0$ are compared with analytical solutions obtained by Chen and Sih [30] for elastic isotropic case (see, Fig. 2) and by Ohyoshi [29] for orthotropic materials (see, Fig. 3). Figure 2 a), b) shows normalized SIFs $K_{I}$ and $K_{I I}$ versus normalized frequency $\Omega$ for normal incident plane time-harmonic P-wave (Fig. 2 a) and for normal incident SVwave (Fig. 2 b) propagating in elastic isotropic plane with a crack. Figure 3 presents normalized SIFs $K_{I}$ versus normalized frequency $\Omega$ for normal incident plane time-harmonic P-wave propagating in elastic 

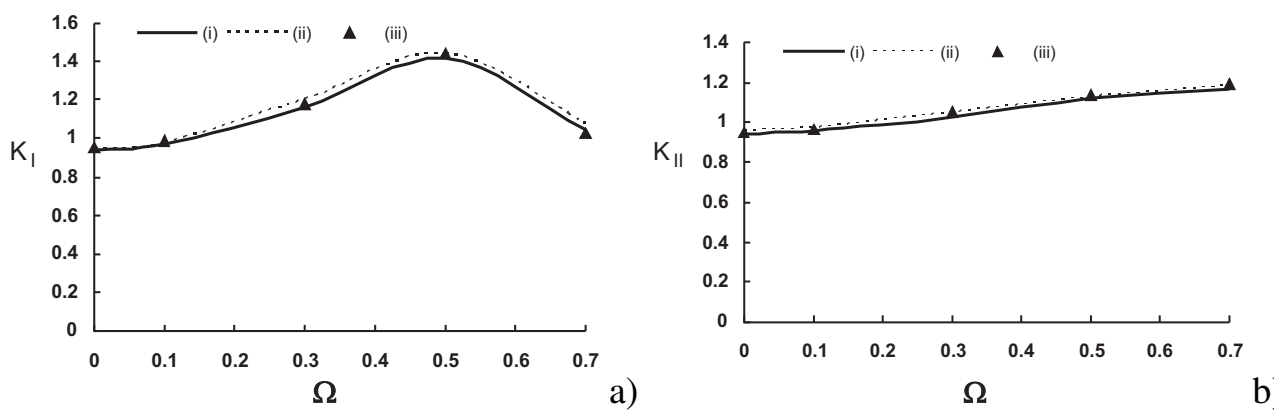

Fig. 2. Comparison for normalized SIFs versus normalized frequency $\Omega$ between (i) authors“ BIEM result for macro-crack; (ii) Chen and Sih [30] analytical result for macro-crack; (iii) authors' BIEM result for blunt nano-crack with $m=0$ : a) $K_{I}$ for normal incident P-wave; $K_{I I}$ for normal incident $\mathrm{SV}$-wave.

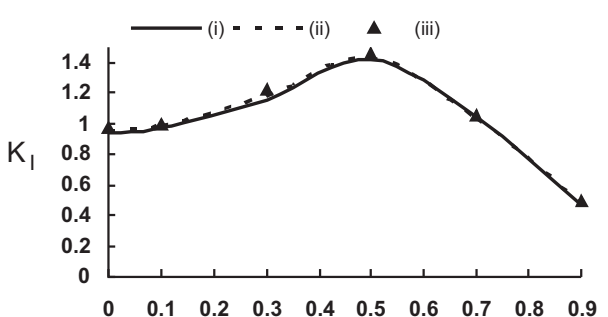

$\Omega$

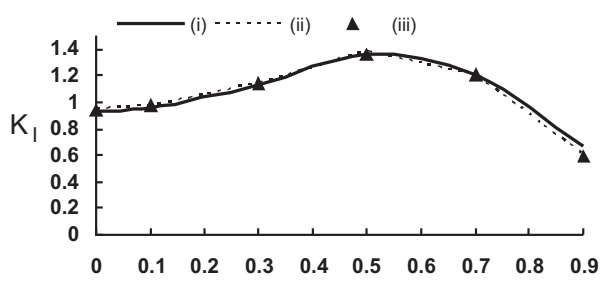

$\Omega$

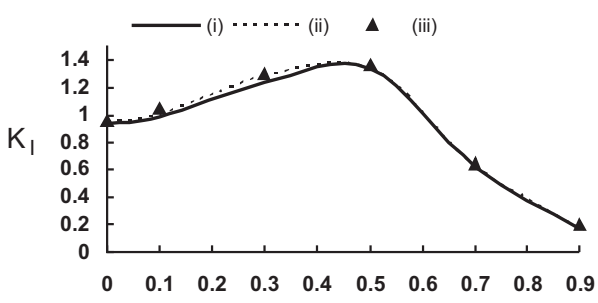

$\Omega$

b)

Fig. 3. Normalized SIF $K_{I}$ versus normalized frequency $\Omega$ for normal incident P-wave, where (i) authors` BIEM result for macro-crack; (ii) Ohyoshi [29] analytical result for macrocrack; (iii) authors' BIEM result for blunt nano-crack with $m=0$ for different orthotropic materials: a) isotropic plane; b) orthotropic plane of material case b); c) orthotropic plane of material case $\mathrm{c}$ ).

orthotropic plane with a crack. Three different materials are used: materials in cases a) - c). The excellent agreement of results in Fig. 2 and Fig. 3 demonstrate that the developed numerical scheme works with a high accuracy and good convergence in the considered frequency interval. 


\subsection{PARAMETRIC STUDY}

The aim of this section is to reveal the sensitivity of the stress concentration fields in cracked anisotropic plane subjected to dynamic time-harmonic loads to the frequency, crack-size, surface effects and type of the material anisotropy. Figures 4 and 5 show the normalized SCF versus normalized frequency $\Omega$ for incident P-wave with incident angle $\varphi=\pi / 2$ (Fig. 4) and for incident SV-wave with incident angle $\varphi=\pi / 3$ (Fig. 5) for different values of surface parameter $m=0.0,0.008,0.04,0.2,1.0$, defined in Section 4.1. It is considered a blunt nano-crack located in: isotropic plane of material case a); orthotropic plane of material case b); orthotropic plane of material case c).

The obtained results in Figs. 4, 5 demonstrate the surface elasticity effect. With increasing of $m$ (respectively decreasing of $b b_{m}$ ), the influence of the surface effect
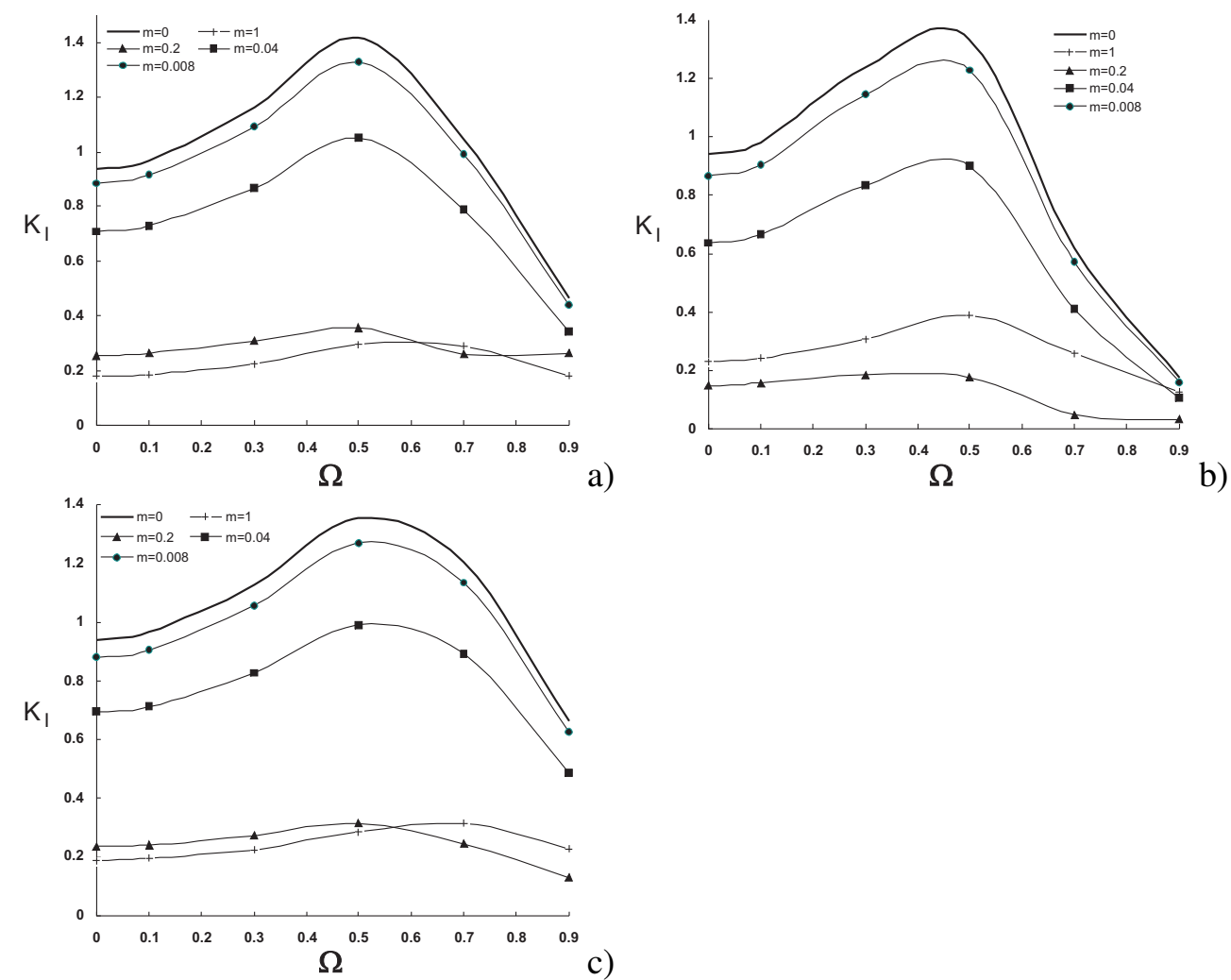

b)

Fig. 4. Normalized SCF $K_{I}$ versus normalized frequency $\Omega$ for normal incident P-wave at different values of surface parameter $m$ for a blunt crack in: a) isotropic plane case a); b) orthotropic plane of material case b); c) orthotropic plane of material case c). 
increases, and as a result the stress concentration field is reduced strongly in the whole considered frequency interval. In the case of $m=0$, the obtained solution recovers those for the macro-crack. These figures reveal the sensitivity of the SCF to the frequency of the dynamic load, to specific surface properties, to material anisotropy and to the size of the blunt crack's root curvature. The above analyses demonstrate and confirm the suggestion that surface elasticity has a considerable impact on the near-tip fields of the crack. Important result is that SIF $K_{I I}$ does not depend on the surface parameter $m$, see Fig. 5 d).
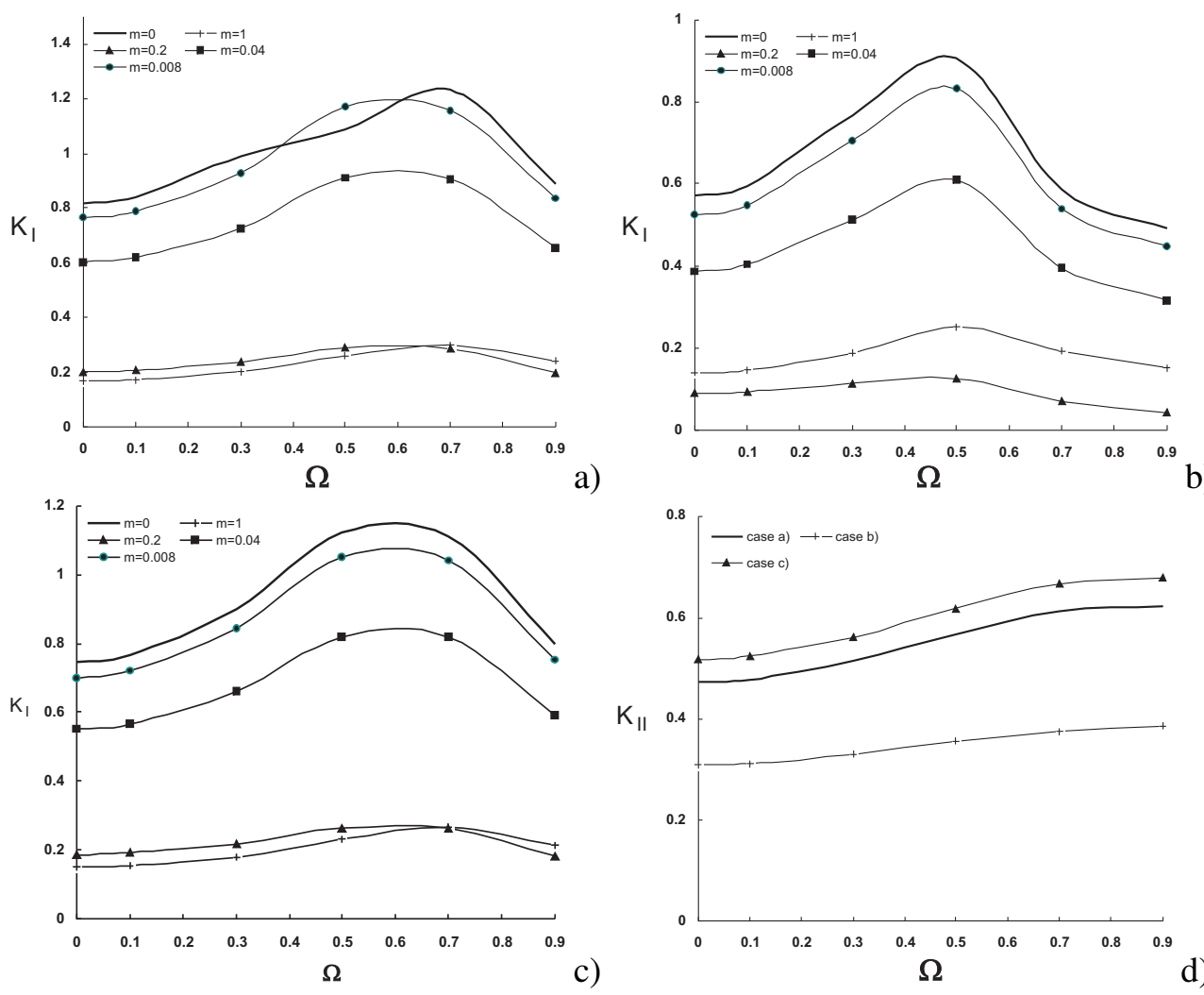

Fig. 5. Normalized SCF $K_{I}$ versus normalized frequency $\Omega$ of incident SV-wave with angle $\varphi=\pi / 3$ for different values of surface parameter $m$ for a blunt crack in: a) isotropic plane; b) orthotropic plane of material case b); c) orthotropic plane of material case c); d) normalized SIF $K_{I I}$ versus normalized frequency $\Omega$ of incident $\mathrm{SV}$-wave with angle $\varphi=\pi / 3$ which is independent of $m$. 


\section{CONCLUSiON}

2D elastodynamic analysis of in-plane finite nano-crack in an anisotropic plane is presented by non-hypersingular traction BIEM in the frequency domain in conjunction with the surface elasticity model of Gurtin and Murdoch [1]. In this model a surface/interface is regarded as an elastic but negligibly thin membrane with the properties that is adhered to the underlying bulk material without slipping and with elastic constants different from the bulk. The equilibrium and constitutive equations in the bulk are the same as those in the classical elasticity theory, but the presence of surface stresses gives rise to non-classical boundary conditions taking into consideration the residual surface tension and surface deformation. A parametric study for local dynamic stress concentration field near nano-cracks in materials with different type of anisotropy is presented. The numerical results show that the wave-nano-crack interaction in anisotropic continua depends on the combined influence of the incident wave frequency, the ratio of the wave length and the crack length, the type of material anisotropy and to the size of blunt crack's root curvature. The decreasing of the radius curvature of the blunt crack to the nano-scale provokes strong reduction of the intensity of the stress concentration field near the crack-tip in the whole considered frequency interval. The influence of the size effect on the mechanical properties of the nano-composites is in the base of the new advanced nano-technologies.

\section{ACKNOWLEDGEMENT}

The authors acknowledge the support of the Bulgarian NSF under the Grant No. DFNI-I 02/12.

\section{REFERENCES}

[1] Gurtin, M. E., A. I. Murdoch. A Continuum Theory of Elastic Material Surfaces. Arch. Ration. Mach. Anal., 57 (1975), 291-323.

[2] Fu, X. L., G. F. WANG, X. Q. FANG. Surface Effects on Mode-I Crack-tip Fields: A Numerical Study. Eng. Fract. Mech., 77 (2010), 1048-1057.

[3] Yang, Q., J. X. Liu, X. Q. Fang. Dynamic Stress in a Semi-infinite Solid with a Cylindrical Nano-inhomogeneity Considering Nanoscale Microstructure. Acta Mech., 223 (2012), 879-888.

[4] Fang, X. Q., L. L. Zhang, J. X. LiU. Dynamic Stress Concentration around Two Interacting Coated Nanowires with Surface/interface Effect. Meccanica, 48 (2013), 287296, DOI: 10.1007/s11012-012-9600-z.

[5] WAng, G. F. Multiple Diffraction of Plane Compression Waves by Two Circular Cylindrical Holes with Surface Effects. J. Appl. Phys., 105 (2009), 013507-1-013507-6.

[6] Zhang, Q. F., G. F. WANG, P. Schiavone. Diffraction of Plane Compression Waves by an Array of Nanosized Cylindrical Holes. ASME, J. Appl. Mech., 78 (2011), 021003$1-021003-5$. 
[7] WAng, G. F., T. J. WAng, X. Q. Feng. Surface Effects on the Diffraction of Plane Compression Waves by a Nanosized Circular Hole. Appl. Phys. Lett., 89 (2006), 231923-1-231923-3.

[8] RU, Y., G. F. WANG, T. J. WANG. Diffraction of Elastic Waves and Stress Concentration near a Cylindrical Nano-inclusion incorporating Surface Effect. J. Vib. Acoust., 13 (2009), No. 6, 061077-061077-7.

[9] Parvanova, S., G. Manolis, P. Dineva. Wave Scattering by Nano-heterogeneities Embedded in an Elastic Matrix via BEM. Eng. Anal. Bound. Elem., 56 (2015), 57-69.

[10] Parvanova, S., G. Vasilev, P. Dineva, G. Manolis. Dynamic Analysis of Nanoheterogeneities in a Finite-sized Solid by Boundary and Finite Element Methods. Int. J. Solids. Str., 80 (2016), 1-18.

[11] Parvanova, S., G. Vasilev, P. Dineva. Elastodynamic Analysis of Anisotropic Elastic Solid with Multiple Nano-cavities. Europ. J. Comput. Mech., 25 (2016), No. 1-2, 129-146.

[12] Shimada, T., K. Ouchi, Y. Chinara, T. Kitamura. Breakdown of Continuum Fracture Mechanics at the Nano-scale, Kyoto University, Technical Report, Dep. Mech. Eng. Sci., 2015.

[13] Sendova, T., J. R. Walton. A New Approach to the Modeling and Analysis of Fracture through Extension of Continuum Mechanics to the Nano-scale. Math. Mech. Solids, 15 (2010), No. 3, 368-413.

[14] Wu, C. H. The Effect of Surface Stress on the Confguration Equilibrium of Voids and Cracks. J. Mech. Phys. Solids, 47 (1999), 2469-2492.

[15] Wang, G. F., Y. Li. Influence of Surface Tension on Mode I Crack Tip Field. Eng. Fract. Mech., 109 (2013), 290-301.

[16] NAN, H., B. WANG. Effect of Residual Surface Stress on the Fracture of Nanoscale Materials. Mech. Res. Commun., 44 (2012), 30-34.

[17] Shenoy, V. B. Atomistic Calculations of Elastic Properties of Metallic FCC Crystal Surfaces. Phys. Rev. B, 71 (2005), No. 9, 094104.

[18] Hoagland, R. G., M. S. Daw, J. P. Hirth. Some Aspects of Forces and Fields in Atomic Models of Crack Tips. J. Mater. Res., 6 (1991), 2565-2571.

[19] Wang, G. F., X. Q. Feng, T. J. Wang, W. GaO. Surface Effects on the Near-tip Stresses for Mode-I and Mode-III Cracks. ASME, J. Appl. Mech., 75 (2008), 0110011-011001-5.

[20] FU, X. L., G. F. WANG, X. Q. FAnG. Surface Effects on the Near-tip Stress Fields of a Mode-II crack. Int. J. Fract., 151 (2008), 95-106.

[21] FU, X. L., G. F. WANG, X. Q. FANG. Effects of Surface Elasticity on Mixed-mode Fracture. Int. J. Appl. Mech., 3 (2011), No. 3, 435-446.

[22] Gao, W., S. W. Yu, G. Y. Huang. Finite Element Characterization of the Sizedependent Mechanical Behaviour in Nano-systems. Nanotechnology, 17 (2006), 1118 1122 . 
[23] Garcia-Sanchez, F. Numerical Study of Fracture Problems in Elastic Anisotropic and Piezoeleztric Solids, PhD Thesis, Spain, Sevilla, University of Sevilla, Engineering High School, 2005.

[24] Voigt, W. Lehrbuch der Kristallphysik, Leipzig, Teubner, 18 (1910), p. 716.

[25] Dineva, P., D. Gross, R. Müller, T. Rangelov. Dynamic Fracture of Piezoelectric Materials. Solutions of Time-harmonic Problems via BIEM, Solid Mechanics and its Applications, Switzerland, Springer Int. Publ., 212 (2014).

[26] Suo, Z., C. Kuo, D. Barnett, J. Willis. Fracture Mechanics for Piezoelectric Ceramics. J. Mech. Phys. Solids, 40 (1992) 739-765.

[27] Dong, C. Y., E. PAN. Boundary Element Analysis of Inhomogeneities of Arbitrary Shapes with Surface and Interface Effects. Eng. Anal. Bound. Elem., 35 (2011), 9961002.

[28] Zhang, C., D. Gross. On Wave Propagation in Elastic Solids with Cracks, Southampton, Comput. Mech. Publ., 1998.

[29] Oнyoshi, T. Effect of Orthotropy on Singular Stresses for a Finite Crack. ASME, J. Appl. Mech., 40 (1973), 491-497.

[30] Chen, E. P., G. C. Sin. Scattering Waves about Stationary and Moving Cracks. In G. C. Sih, editor, Mechanics of Fracture: Elastodynamic Crack Problems, Leyden, Noordhoff, 19 (1977), 119-212. 